Received: 17.08 .2020

Revised: 24.09 .2020

Accepted: 16.10 .2020

DOI: $10.17804 / 2410-9908.2020 .5 .026-044$

\title{
ON NUMERICAL METHODS FOR CONSTRUCTING BENCHMARK SOLUTIONS TO A NONLINEAR HEAT EQUATION WITH A SINGULARITY
}

\author{
A. L. Kazakov ${ }^{1, a)}$, L. F. Spevak ${ }^{1, b)^{*}}$, E. L. Spevak ${ }^{2, c)}$ \\ ${ }^{1}$ Institute of Engineering Science, Ural Branch of the Russian Academy of Sciences, \\ 34 Komsomolskaya St., Ekaterinburg, Russian Federation \\ ${ }^{2}$ Institute of Natural Sciences and Mathematics, B. N. Yeltsin Ural Federal University, \\ 48 Kuibysheva St., Ekaterinburg, 620000, Russian Federation \\ a) iD http://orcid.org/0000-0002-3047-1650 a_kazakov@mail.ru; \\ b) iD http://orcid.org/0000-0003-2957-6962 ه1fs@imach.uran.ru; \\ c) spevak155@gmail.com \\ *Corresponding author. E-mail: 1fs@imach.uran.ru \\ Address for correspondence: ul. Komsomolskaya, 34, Ekaterinburg, 620049, Russian Federation \\ Tel.: +7 (343) 36230 22; fax: +7 (343) 3745330
}

The paper deals with the construction of exact solutions to a nonlinear heat equation with degeneration in the case of the zero value of the required function. Generically self-similar solutions and traveling wave solutions are considered, the construction of which reduces to solving Cauchy problems for a nonlinear second-order ordinary differential equation with a singularity before the higher derivative. Two approaches are proposed to solve the Cauchy problems: the analytical solution by the power series method and the numerical solution by the boundary element method on a specified segment. A complex computational experiment is carried out to compare the above two methods with each other and with the finite difference methods, namely the Euler method and the fourth-order Runge-Kutta method. Power series segments are used on the first step of the finite difference solutions in order to resolve the singularity. The comparison of the application domains, the accuracy of the solutions and their dependence on the parameters of a certain problem shows that the boundary element method is the most universal, although not the most accurate for some particular examples. The conclusions drawn allow us to construct benchmark solutions to verify the approximate solutions of the nonlinear heat equation by various methods in a wide range of parameter values.

Keywords: nonlinear heat equation, exact solution, generically self-similar solution, traveling wave, power series, boundary element method.

\section{Acknowledgment}

The work was supported by the RFBR, project No. 20-07-00407.

\section{References}

1. Vazquez J.L. The Porous Medium Equation: Mathematical Theory, Oxford, Clarendon Press, 2007, 648 p. ISBN-10: 0198569033, ISBN-13: 978-019856903.

2. Samarskii A.A., Galaktionov V.A., Kurdyumov S.P., Mikhailov A.P. Blow-up in Quasilinear Parabolic Equations, NY, Berlin, Walter de Gruyte, 1995, 534 p. ISBN 3-11-012754-7.

3. Zel'dovich Ya.B., Kompaneets A.S. K teorii rasprostraneniya tepla pri teploprovodnosti, zavisyashchey ot temperatury [Towards a theory of heat conduction with thermal conductivity 
depending on the temperature]. In: Collection of Papers Dedicated to 70th Anniversary of A.F. Ioffe, Moscow, Akad. Nauk SSSR Publ., 1950, pp. 61-71. (In Russian).

4. Barenblatt G.I., Vishik I.M. On the Final Velocity of Propagation in Problems of Nonstationary Filtration of Liquid and Gas. Prikladnaya matematika i mekhanika, 1956, vol. 20, no. 3, pp. 411-417. (In Russian).

5. Oleynik O.A., Kalashnikov A.S. Chzhou Yuy-Lin. The Cauchy Problem and Boundary Value Problems for Equations of the type of Non-stationary Filtration. Izvestiya AN SSSR. Seriya matematicheskaya, 1958, vol. 22, no. 5, pp. 667-704. (In Russian).

6. $\quad$ Sidorov A.F. In: Izbrannye Trudy: Matematika. Mekhanika [Selected Works: Mathematics. Mechanics]. Moscow, Fizmatlit Publ., 2001, 576 p. (In Russian). ISBN 5-9221-0103-X.

7. Kazakov A.L., Lempert A.A. Analytical and Numerical Studies of the Boundary Value Problem of a Nonlinear Filtration with Degeneration. Vychislitelnye tekhnologii, 2012, vol. 17, no. 1, pp. 57-68. (In Russian).

8. Kazakov A.L., Spevak L.F. Boundary Elements Method and Power Series Method for One-dimensional Nonlinear Filtration Problems. Izvestiya IGU. Seriya Matematika [The Bulletin of Irkutsk State University. Series Mathematics], 2012, vol. 5, no. 2, pp. 2-17. (In Russian).

9. Kazakov A.L., Spevak L.F. Numerical and analytical studies of a nonlinear parabolic equation with boundary conditions of a special form. Applied Mathematical Modelling, 2013, vol. 37, iss. 10-11, pp. 6918-6928. DOI: 10.1016/j.apm.2013.02.026.

10. Kazakov A.L., Kuznetsov P.A., Spevak L.F. On a Degenerate Boundary Value Problem for the Porous Medium Equation in Spherical Coordinates. Trudy Instituta Matematiki i Mekhaniki UrO RAN, 2014, vol. 20, no. 1, pp. 119-129. (In Russian).

11. Kazakov A.L., Spevak L.F. An analytical and numerical study of a nonlinear parabolic equation with degeneration for the cases of circular and spherical symmetry. Applied Mathematical Modelling, 2015, vol. 40, iss. 2, pp. 1333-1343. DOI: 10.1016/j.apm.2015.06.038.

12. Spevak L.F., Kazakov A.L. Constructing numerical solutions to a nonlinear heat conduction equation with boundary conditions degenerating at the initial moment of time. In: AIP Conference Proceedings, 2016, vol. 1785, iss. 1, pp. 040076. DOI: 10.1063/1.4967136

13. Kazakov A.L., Kuznetsov P.A., Spevak L.F. A heat wave problem for a degenerate nonlinear parabolic equation with a specified source function. In: AIP Conference Proceedings, 2018, vol. 2053, pp. 030024. Available at: https://doi.org/10.1063/1.5084385

14. Kazakov A.L., Nefedova O.A., Spevak L.F. Solution of the Problem of Initiating the Heat Wave for a Nonlinear Heat Conduction Equation Using the Boundary Element Method. Computational Mathematics and Mathematical Physics, 2019, vol. 59, no. 6, pp. 1015-1029. DOI: $10.1134 / \mathrm{S} 0965542519060083$.

15. Kazakov A.L. On exact solutions to a heat wave propagation boundary-value problem for a nonlinear heat equation. Sibirskiye Elektronnye Matematicheskiye Izvestiya [Siberian Electronic Mathematical Reports], 2019, vol. 16, pp. 1057-1068. (In Russian). DOI: 10.33048/semi.2019.16.073. Available at: http://semr.math.nsc.ru/v16/p1057-1068.pdf

16. Spevak L.F., Kazakov A.L. Solving a degenerate nonlinear parabolic equation with a specified source function by the boundary element method. In: AIP Conference Proceedings, 2017, vol. 1915, pp. 040054. Available at: https://doi.org/10.1063/1.5017402

17. Kazakov A.L., Spevak L.F., Nefedova O.A. On the Numerical-Analytical Approaches to Solving a Nonlinear Heat Conduction Equation with a Singularity. Diagnostics, Resource and Mechanics of materials and structures, 2018, iss. 6, pp. 100-116. DOI: 10.17804/24109908.2018.6.100-116. Available at: http://dream-journal.org/issues/2018-6/2018-6_232.html

18. Banerjee P.K., Butterheld R. Boundary element methods in engineering science, US, McGraw-Hill Inc., 1981, 452 p. ISBN-10: 0070841209, ISBN-13: 978-0070841208. 
19. Nardini D., Brebbia C.A. A New Approach to Free Vibration Analysis using Boundary Elements. Applied Mathematical Modelling, 1983, vol. 7, no. 3, pp. 157-162. DOI: 10.1016/0307904X(83)90003-3.

20. Wrobel L.C., Brebbia C.A. Nardini D. The dual reciprocity boundary element formulation for transient heat conduction. In: Finite elements in water resources VI, Springer-Verlag, Berlin, Germany, 1986, pp. 801-811.

21. Tanaka M., Matsumoto T., Yang Q.F. Time-stepping boundary element method applied to 2-D transient heat conduction problems. Applied Mathematical Modelling, 1994, vol. 18, pp. 569-576. DOI: 10.1016/0307-904X(94)90142-2.

22. Tanaka M., Matsumoto T., Takakuwa S. Dual reciprocity BEM for time-stepping approach to the transient heat conduction problem in nonlinear materials. Computer Methods in Applied Mechanics and Engineering, 2006, vol. 195, pp. 4953-4961. DOI: 10.1016/j.cma.2005.04.025.

23. Divo E., Kassab A.J. Transient non-linear heat conduction solution by a dual reciprocity boundary element method with an effective posteriori error estimator. Computers, Materials and Continua, 2005, vol. 2, pp. 277-288. DOI: 10.3970/cmc.2005.002.277.

24. Wrobel L.C., Brebbia C.A. The dual reciprocity boundary element formulation for nonlinear diffusion problems. Computer Methods in Applied Mechanics and Engineering, 1987, vol. 65, pp. 147-164. DOI: 10.1016/0045-7825(87)90010-7.

25. Singh K.M., Tanaka M. Dual reciprocity boundary element analysis of transient advectiondiffusion. International Journal of Numerical Methods for Heat \& Fluid Flow, 2003, vol. 13, pp. 633-646. DOI: 10.1108/09615530310482481.

26. Al-Bayati S.A., Wrobel L.C. A novel dual reciprocity boundary element formulation for twodimensional transient convection-diffusion-reaction problems with variable velocity. Engineering Analysis with Boundary Elements, 2018, vol. 94, pp. 60-68. DOI: 10.1016/j.enganabound.2018.06.001.

27. Fendoglu H., Bozkaya C., Tezer-Sezgin M. DBEM and DRBEM solutions to 2D transient convection-diffusion-reaction type equations. Engineering Analysis with Boundary Elements, 2018, vol. 93, pp. 124-134. DOI: 10.1016/j.enganabound.2018.04.011.

28. Powell M.J.D. The Theory of Radial Basis Function Approximation. In: W. Light, ed. Advances in Numerical Analysis, Oxford Science Publications, Oxford, UK, 1992, vol. 2.

29. Golberg M.A., Chen C.S., Bowman H. Some recent results and proposals for the use of radial basis functions in the BEM. Engineering Analysis with Boundary Elements, 1999, vol. 23, pp. 285-296. DOI: 10.1016/S0955-7997(98)00087-3.

30. Kazakov A.L., Kuznetsov P.A., Spevak L.F. Analytical and numerical construction of heat wave type solutions to the nonlinear heat equation with a source. Journal of Mathematical Sciences, 2019, vol. 239, no. 2, pp. 111-122. DOI 10.1007/s10958-019-04294-x.

31. Kazakov A.L., Spevak L.F. Numerical Study of Travelling Wave Type Solutions for the Nonlinear Heat Equation. In: AIP Conference Proceedings, 2019, vol. 2176, pp. 030006. DOI: $10.1063 / 1.5135130$.

32. Polyanin A.D., Zaytsev V.F., Zhurov A.I. Nelineynye uravneniya matematicheskoy fiziki $i$ mekhaniki. Metody resheniya [A Nonlinear Equations of Mathematical Physics and Mechanics. Solution Methods]. Moscow, Yurayt Publ., 2017, 256 p. (In Russian). ISBN 978-5-534-02317-6. 
Подана в журнал: 17.08.2020

УДК 517.958:519.633

DOI: $10.17804 / 2410-9908.2020 .5 .026-044$

\title{
О ЧИСЛЕННЫХ МЕТОДАХ ПОСТРОЕНИЯ ЭТАЛОННЫХ РЕШЕНИЙ ДЛЯ НЕЛИНЕЙНОГО УРАВНЕНИЯ ТЕПЛОПРОВОДНОСТИ С ОСОБЕННОСТЬЮ
}

\author{
А. Л. Казаков ${ }^{1, \text { a) }}$, Л. Ф. Спевак ${ }^{1, \text { б)* }}$ Е. Л. Спевак ${ }^{2, \text { в) }}$ \\ ${ }^{1}$ Федеральное государственное бюджетное учреждение науки \\ Институт машиноведения Уральского отделения Российской академии наук, \\ 34, ул. Комсомольская, г. Екатеринбург, 620049, Российская Федерация \\ ${ }^{2}$ Институт естественных наук и математики Уральского Федерального университета \\ имени первого Президента России Б. Н. Ельциина, \\ 48, ул. Куйбышева, г. Екатеринбург, 620000, Российская Федерация \\ a) iD http://orcid.org/0000-0002-3047-1650 هa_kazakov@mail.ru; \\ б) (iD http://orcid.org/0000-0003-2957-6962 ه1fs@imach.uran.ru; \\ в) spevak155@gmail.com \\ *Ответственный автор. Электронная почта: lfs@imach.uran.ru \\ Адрес для переписки: ул. Комсомольская, 34, 620049, Екатеринбург, Российская Федерация \\ Тел.: +7 (343) 362-30-22; факс: +7 (343) 374-53-30
}

Работа посвящена построению точных решений нелинейного уравнения теплопроводности с вырождением в случае нулевого значения искомой функции. Рассмотрены обобщенноавтомодельные решения и решения типа бегущей волны, нахождение которых сводится к решению задач Коши для обыкновенного дифференциального уравнения второго порядка с особенностью при старшей производной. Для решения этих задач предложены два подхода: построение аналитического решения методом степенных рядов и построение решения на заданном отрезке методом граничных элементов. В проведенном объемном вычислительном эксперименте построенные двумя методами решения сравнивались между собой, а также с разностными решениями методами Эйлера и Рунге-Кутта, в которых для раскрытия особенностей на первом шаге используются отрезки степенных рядов. Сопоставление областей применимости методов, точности решений и их зависимости от параметров конкретной задачи показало набольшую универсальность метода граничных элементов, хотя для отдельных примеров лучшую точность имели решения другими методами. Сделанные выводы позволят с достаточной корректностью строить эталонные решения для верификации приближенных решений нелинейного уравнения теплопроводности различными методами в широком диапазоне значений параметров.

Ключевые слова: нелинейное уравнение теплопроводности, точное решение, обобщенноавтомодельное решение, бегущая волна, степенной ряд, метод граничных элементов.

\section{1. Введение}

Верификация численных методов решения краевых задач для дифференциальных уравнений с частными производными является важной составляющей их разработки. Особую актуальность указанная проблематика приобретает в связи с исследованием нелинейных уравнений с особенностями, поскольку в этом случае очень редко удается доказать сходимость метода и тогда в основе верификации обычно лежит сравнение полученных решений с известными точными решениями, либо с приближенными решениями, полученными другими методами. Статья посвящена исследованию методов построения точных решений типа бегущей волны, а также обобщенно-автомодельных решений для вырождающегося нелиней- 
ного параболического уравнения, которые авторы используют для верификации разрабатываемых ими алгоритмов на основе метода граничных элементов (МГЭ).

Рассмотрим нелинейное уравнение теплопроводности с источником [1, 2]:

$$
T_{t}=\left(k(T) T_{x}\right)_{x}+H(T) .
$$

Здесь $t$ - время; $x$ - пространственная координата; $T$ - искомая функция (температура); $k(T)$ - функция источника. Будем считать, что $k(0)=0 ; H(0)=0$. В случае монотонности и достаточной гладкости функции $k(T)$ подстановкой $u=k(T)$ уравнение (1.1) приводим к виду

$$
u_{t}=u u_{x x}+F(u) u_{x}^{2}+h(u)
$$

Здесь $F(u)=\frac{u \varphi^{\prime \prime}(u)}{\varphi^{\prime}(u)}+1 ; h(u)=\frac{H(\varphi(u))}{\varphi^{\prime}(u)} ; T=\varphi(u)-$ функция, обратная к $u=k(T)$.

Важным свойством уравнения (1.2) является вырождение параболического типа при $u=0$, что приводит к существованию решений, имеющих вид тепловой волны, распространяющейся по холодному (нулевому) фону с конечной скоростью [3-6]. Именно верификации численного метода построения таких решений посвящена настоящая работа.

Данное исследование продолжает большой цикл работ авторов, посвященных построению и исследованию решений, имеющих тип тепловой волны. Ранее авторы исследовали уравнение (1.2) при заданном законе движения нулевого фронта тепловой волны

$$
\left.u\right|_{x=a(t)}=0
$$

а также задачи об инициировании тепловой волны при краевом режиме в неподвижной точке

$$
\left.u\right|_{x=0}=f(t)
$$

и в подвижной точке,

$$
\left.u\right|_{x=g(t)}=f(t)
$$

Легко видеть, что условия (1.3) и (1.4) являются частными случаями (1.5). Для степенной функции $k(T)$ были доказаны теоремы существования и единственности аналитического решения в различных постановках задачи (1.2), (1.5) [7-14]. Для произвольного вида функции $k(T)$ аналогичная теорема была доказана для задачи (1.2), (1.3) [15]. Другим направлением исследования была разработка численных алгоритмов решения всех этих задач на заданном конечном промежутке времени: задача (1.2), (1.3) в различных постановках была рассмотрена в работах $[8,9,11,13,16]$, задача $(1.2),(1.4)$ - в $[8,9,11,12,14]$, задача $(1.2),(1.5)[9,11]$. Подробный обзор подходов авторов к численным и аналитическим исследованиям задач для уравнения (1.2) приведен в статье [17].

В основе численных алгоритмов, разработанных авторами, лежит решение с помощью МГЭ [18] и метода двойственной взаимности (МДВ) [19]. Подобный подход, впервые предложенный для решения нестационарных задач теплопроводности в работе [20], является эффективным средством решения краевых задач для параболических уравнений различного вида: теплопроводности [21-23], диффузии [24], адвекции-диффузии [25], конвекции-реакции-диффузии $[26,27]$ и др. Общим подходом в этих работах является представ- 
ление параболического уравнения в виде неоднородного уравнения Пуассона с производной по времени в правой части и применение МГЭ для решения полученного эллиптического уравнения. Важную роль при использовании МДВ играет выбор вида радиальных базисных функций $[28,29]$. При решении нелинейных задач методом граничных элементов [22-24] МДВ позволяет свести интегралы по области к интегралам по границе, что позволяет снизить размерность задачи, и дальнейшее решение определяется выбранной дискретизацией по времени.

Несмотря на обоснованность и широкое использование применяемого подхода к решению разработанные алгоритмы необходимо верифицировать сравнением с известными точными решениями. Авторами были рассмотрены различные подходы к их построению: исследовались обобщенно-автомодельные решения, имеющие тип тепловой волны $[15,30]$, а также решения типа бегущей волны [31]. Общим в этих подходах является то, что построение решения сводится к задаче Коши для обыкновенного дифференциального уравнения (ОДУ) второго порядка с особенностью при старшей производной. Получить решение такой задачи в конечном аналитическом виде удается лишь в единичных случаях. Однако найденное с достаточной точностью приближенное решение задачи Коши также позволит получить эталонное решение для тестирования численного решения исходной задачи для уравнения (1.2). Отсюда вытекает основная цель данного исследования: провести сравнительную оценку точности различных численных алгоритмов решения задач Коши, возникающих при построении точных решений уравнения (1.2), и определить наиболее подходящий метод.

\section{2. Обобщенно-автомодельные решения}

Рассмотрим случай, когда $k(T)=T^{\sigma}$ и $h(u)=\alpha u^{\beta}$. Тогда задача (1.2), (1.3) может быть записана следующим образом:

$$
u_{t}=u u_{x x}+\frac{1}{\sigma} u_{x}^{2}+\alpha u^{\beta},\left.u\right|_{x=a(t)}=0 .
$$

Будем искать решения задачи (2.1) в виде

$$
u=\lambda(t) w(z), z=1-\frac{x}{a(t)}
$$

Решения подобного типа будем называть обобщенно-автомодельными [32]. Подставив (2.2) в (2.1), после приведения подобных слагаемых и умножения обеих частей на $a^{2}(t) / \lambda^{2}(t)$ получим:

$$
\begin{gathered}
w w^{\prime \prime}+\frac{1}{\sigma}\left(w^{\prime}\right)^{2}+\frac{a(t) a^{\prime}(t)}{\lambda(t)}(z-1) w^{\prime}-\frac{a^{2}(t) \lambda^{\prime}(t)}{\lambda^{2}(t)} w+\frac{\alpha a^{2}(t)}{\lambda^{2-\beta}(t)} w^{\beta}=0, \\
w(0)=0 .
\end{gathered}
$$

Утвержжение. Уравнение (2.3) является обыкновенным дифференциальным относительно искомой функиии $w(z)$, если закон движения фронта тепловой волны а $(t)$ является при согласованном значении $\beta$ либо экспонентой $(\beta=1)$, либо степенной функцией.

Доказательство. Можно видеть, что (2.3) становится обыкновенным дифференциальным уравнением (ОДУ), когда выполнены равенства 


$$
\frac{a(t) a^{\prime}(t)}{\lambda(t)}=A=\text { const } ; \frac{a^{2}(t) \lambda^{\prime}(t)}{\lambda^{2}(t)}=B=\text { const } ; \frac{a^{2}(t)}{\lambda^{2-\beta}(t)}=D=\text { const }
$$

Проинтегрировав систему (2.5), можно убедиться, что, если $B / A=2, \beta=1$, решением является экспонента $a(t)=C_{1} e^{C_{2} z}$; если же $B / A \neq 2,-$ степенная функция $a(t)=\left(C_{3} t+C_{4}\right)^{\omega}$. Здесь $C_{i}, i=1, \ldots, 4 ; \omega=(\beta-2) /(2 \beta-2)$ - константы, $\beta \neq 1,2$. Утверждение доказано.

Не теряя общности рассмотрения, можно принять, что $A=1$. При выполнении условий (2.5) задача (2.3), (2.4) может быть записана в виде

$$
w w^{\prime \prime}+\frac{1}{\sigma}\left(w^{\prime}\right)^{2}+(z-1) w^{\prime}-B w+\alpha D w^{\beta}=0, w(0)=0,
$$

где $B=2 /(\beta-2) ; D=1 / C_{2}$ в случае показательного закона движения фронта тепловой волны; $D=\left(C_{3} \omega\right)^{\beta-2}$ в случае степенного закона движения. В соответствии с физическим смыслом задачи предположим, что $w^{\prime}(0)>0$. Тогда условием совместности задачи (2.6) является равенство

$$
w^{\prime}(0)=\sigma
$$

Задача Коши (2.6), (2.7) имеет особенность при $z=0$ и для нее неприменимы классические теоремы существования и единственности. Существование и единственность аналитического решения (2.6), (2.7) при натуральных значениях $\beta$ следует из ранее полученных результатов авторов [15].

Таким образом, с учетом равенства $\lambda(t)=a(t) a^{\prime}(t)(2.5)$, получены следующие обобщенно-автомодельные решения задачи (2.1):

$$
u=\left\{\begin{array}{l}
C_{1}^{2} C_{2} e^{2 C_{2} t} w(z), z=1-\frac{x}{C_{1} e^{C_{2} t}}, \beta=1 \\
C_{3} \omega\left(C_{3} t+C_{4}\right)^{2 \omega-1} w(z), z=1-\frac{\rho}{\left(C_{3} t+C_{4}\right)^{\omega}}, \omega=\frac{\beta-2}{2 \beta-2}, \beta \neq 1,2 .
\end{array}\right.
$$

Здесь функция $w(z)$ удовлетворяет задаче Коши (2.6), (2.7).

\section{3. Бегущая волна}

Рассмотрим теперь случай нелинейности общего вида. Будем искать решение задачи $(1.2),(1.3)$ типа бегущей волны. Представим решение в виде $u(t, x)=v(z)$, где $z=\mu t-x$. Подставляя принятый вид решения в уравнение (1.2) и граничное условие (1.3), приходим к следующей задаче Коши:

$$
\begin{gathered}
v v^{\prime \prime}+F(v)\left(v^{\prime}\right)^{2}-\mu v^{\prime}+h(v)=0 ; \\
v(0)=0, v^{\prime}(0)=\frac{\mu}{F(0)} .
\end{gathered}
$$


Напомним, что $h(0)=0$. Из (3.2) имеем, что $F(0) \neq 0$. Как и в предыдущем разделе, классические теоремы существования и единственности здесь неприменимы. Из ранее полученных результатов авторов [15] следует, что в случае, если функции $F(v)$ и $h(v)$ аналитические в окрестности точки $v=0$ и $F(0)>0$, задача (3.1), (3.2) имеет единственное аналитическое решение, которое в свою очередь порождает решение $u(t, x)=v(\mu t-x)$ задачи (1.2), (1.3) при $a(t)=\mu t$.

Решение задачи (3.1), (3.2), как и задачи (2.6), (2.7), в конечной форме можно получить лишь в редких отдельных случаях. Некоторые из них будут приведены в разд. 6. Однако имеет смысл рассмотреть возможность построения приближенных решений этих задач аналитическими и численными методами.

\section{4. Решение методом степенных рядов}

Рассмотрим задачу Коши для ОдУ следующего вида:

$$
\begin{gathered}
v v^{\prime \prime}+F_{0}\left(v^{\prime}\right)^{2}-\mu v^{\prime}+f(v)\left(v^{\prime}\right)^{2}+g(z) v^{\prime}+h(v)=0 ; \\
v(0)=0 ; v^{\prime}(0)=\frac{\mu}{F_{0}},
\end{gathered}
$$

где $F_{0}>0, \mu>0$ - константы; функции $f, g, h$ являются аналитическими в некоторой окрестности нуля и $f(0)=g(0)=h(0)=0$. Можно убедиться, что частными случаями задачи (4.1), (4.2) являются как задача (2.6), (2.7), так и задача (3.1), (3.2).

Будем строить решение задачи (4.1), (4.2) в виде ряда по степеням $z$ :

$$
v=\sum_{k=0}^{\infty} \frac{v_{k} z^{k}}{k !} ; v_{k}=\left.\frac{d^{k} v}{d z^{k}}\right|_{z=0} \text {. }
$$

При этом $v_{0}=0$ и $v_{1}=\mu / F_{0}$ известны из условия (4.2) и образуют базу индукции. Пусть найдены коэффициенты ряда (4.3) до номера $k$ включительно. Для нахождения $v_{k+1}$ продифференцируем обе части (4.1) $k$ раз по $z$ и положим $z=0$. Получим, что

$$
\mu\left(\frac{k}{F_{0}}+1\right) v_{k+1}=F_{k} .
$$

Конкретный вид правой части (4.4) не приводится из-за громоздкости, однако $F_{k}$ будет зависеть от величин, которые известны в силу предположения индукции. Поскольку $F_{0}>0, \mu \neq 0$, то $v_{k+1}$ отсюда определяется однозначно.

Сходимость ряда (4.3) доказывается методом мажорант по стандартной процедуре $[15,30]$. Его отрезки могут быть использованы для проверки корректности вычислений, которые выполняются другими приближенными методами. Однако здесь необходимо учитывать, что сходимость является локальной, причем размер окрестности, как правило, невелик. 


\section{5. Решение методом граничных элементов}

Обобщая результаты разделов 2 и 3, можно сказать, что при отыскании для уравнения (1.2) точных решений рассмотренных типов необходимо решить задачу Коши вида

$$
v v^{\prime \prime}=G\left(z, v, v^{\prime}\right) ; v(0)=0 ; v^{\prime}(0)=v_{*}^{\prime},
$$

где $G\left(z, v, v^{\prime}\right)$ - известная функция; $v_{*}^{\prime}$ - известное число. В случае, когда задача (5.1) не интегрируется в квадратурах, она может быть решена приближенно. Для построения решения уравнения (1.2) на некотором промежутке времени $t \in\left[0, t_{*}\right]$ задача (5.1) должна быть решена на соответствующем отрезке $z \in\left[0, L\left(t_{*}\right)\right]$. Найденное с достаточной точностью решение может быть использовано для построения эталонного решения уравнения (1.2).

Для решения задачи (5.1) на отрезке $z \in[0, L]$ методом граничных элементов представим ее в следующем виде:

$$
\begin{gathered}
v^{\prime \prime}=\frac{1}{v} G\left(z, v, v^{\prime}\right), z \in(0, L) ; \\
\left.v\right|_{z=0}=0 ; \\
\left.q\right|_{z=0}=-v_{*}^{\prime} .
\end{gathered}
$$

Здесь $q$ - тепловой поток в граничных точках; $q(0)=-v^{\prime}(0) ; q(L)=v^{\prime}(L)$.

Итерационная процедура решения задачи (5.2)-(5.4) с помощью МГЭ при начальном приближении $v_{0}(\xi) \equiv 0$ имеет вид:

$$
\begin{gathered}
v_{k}(\xi)=-v_{*}^{\prime} u^{*}(\xi, 0)+q_{L}^{(k)} u^{*}(\xi, L)-v_{L}^{(k)} q^{*}(\xi, L)-\int_{0}^{L} \frac{1}{v_{k-1}(z)} G\left(z, v_{k-1}(z), v_{k-1}^{\prime}(z)\right) u^{*}(\xi, z) d z \\
\xi \in(0, L),
\end{gathered}
$$

где $u^{*}(\xi, z)=\frac{1}{2}(L-|z-\xi|)-$ фундаментальное решение уравнения Лапласа, $q^{*}(\xi, 0)=-\frac{1}{2} \operatorname{sgn}(\xi)$, $q^{*}(\xi, 0)=\frac{1}{2} \operatorname{sgn}(L-\xi)[18] ; v_{L}^{(k)}=v_{k}(L)$ и $q_{L}^{(k)}=q_{k}(L)=v_{k}^{\prime}(L)-k$-е итерации граничных значений искомой функции и потока, которые являются решением системы граничных интегральных уравнений:

$$
\left\{\begin{array}{l}
v_{L}^{(k)}=v_{*}^{\prime}+2 \int_{0}^{L} \frac{1}{v_{k-1}(z)} G\left(z, v_{k-1}(z), v_{k-1}^{\prime}(z)\right) u^{*}(0, z) d z, \\
v_{L}^{(k)}-q_{L}^{(k)} L=-2 \int_{0}^{L} \frac{1}{v_{k-1}(z)} G\left(z, v_{k-1}(z), v_{k-1}^{\prime}(z)\right) u^{*}(L, z) d z .
\end{array}\right.
$$


Интегралы в правых частях соотношений (5.5) и (5.6) вычисляются с помощью МДВ через разложение по радиальным базисным функциям (РБФ):

$$
\frac{1}{v_{k}(z)} G\left(z, v_{k}(z), v_{k}^{\prime}(z)\right)=\sum_{i=1}^{m} \alpha_{i}^{(k)} f_{i}(z)
$$

Здесь $f_{i}(z)=f\left(\left|z-z_{i}\right|\right)$ - РБФ; $z_{1}, \ldots, z_{m}$ - точки коллокации, расположенные на отрезке $[0, L]$. Для каждой $f_{i}(z)$ существует функция $\hat{u}_{i}(z)$, такая что $\frac{d^{2} \hat{u}_{i}(z)}{d z^{2}}=f_{i}(z)$. Подстановка (5.7) в интеграл приводит его к следующему виду:

$$
\begin{gathered}
\int_{0}^{L} \frac{1}{v_{k}(z)} G\left(z, v_{k}(z), v_{k}^{\prime}(z)\right) u^{*}(\xi, z) d z= \\
=\sum_{i=1}^{m} \alpha_{i}^{(k)}\left[-\hat{u}_{i}(\xi)+\hat{q}_{i}(0) u^{*}(\xi, 0)+\hat{q}_{i}(L) u^{*}(\xi, L)-\hat{u}_{i}(0) q^{*}(\xi, 0)-\hat{u}_{i}(L) q^{*}(\xi, L)\right],
\end{gathered}
$$

где $\hat{q}_{i}(\xi, 0)=-\frac{\partial \hat{u}_{i}(\xi, 0)}{\partial z} ; \hat{q}_{i}(\xi, L)=\frac{\partial \vec{u}_{i}(\xi, L)}{\partial z}$, а коэффициенты $\alpha_{i}^{(k)}$ определяются из системы уравнений, получающейся из (5.7) при $z=z_{i} ; i=1, \ldots, m$.

Итерационный процесс останавливается при $\left|\frac{v_{L}^{(k)}-v_{L}^{(k-1)}}{v_{L}^{(k)}}\right|<\varepsilon$, где $\varepsilon-$ заданный параметр точности. Тогда $v_{k}(\xi)$ будет непрерывно дифференцируемым приближенным решением задачи (5.1) на интервале $\xi \in(0, L)$, которое позволит построить эталонное решение уравнения (1.2).

Отдельно стоит отметить случай, когда искомая функция $v(z)$ обращается в ноль в точке $z=L$ и имеет в этой точке бесконечную производную. Тогда решение имеет максимум $v_{\max }$ в некоторой точке $z_{\max } \in(0, L)$. Значение $L$ при этом неизвестно. Корректно решить такую задачу с помощью представленного алгоритма невозможно, поэтому был разработан специальный подход к решению, включающий в себя два этапа. На первом этапе решается задача (5.2) - (5.4) на отрезке $z \in\left[0, L_{1}\right]$, где значение $L_{1}$ подобрано таким образом, что $z_{\max }<L_{1}<L$. Решение задачи проводится с помощью итерационной процедуры (5.5), (5.6). На втором этапе решается задача для функции $z(v)$, обратной к $v(z)$, соответствующая задаче (5.2)-(5.4) на отрезке $z \in\left[L_{1}, L\right]$ :

$$
\begin{gathered}
z^{\prime \prime}=F\left(v, z, z^{\prime}\right), v \in\left(0, L_{2}\right) ; \\
\left.z\right|_{v=L_{2}}=L_{1} ; \\
\left.q_{z}\right|_{z=L_{2}}=\frac{1}{q\left(L_{1}\right)} .
\end{gathered}
$$


Здесь $L_{2}=v\left(L_{1}\right) ; q_{z}$ - поток для функции $z(v) ; v\left(L_{1}\right)$ и $q\left(L_{1}\right)$ - значения, найденные на первом этапе решения. Задача (5.2)-(5.4) может быть решена итерационным МГЭ аналогично решению на первом этапе. В результате мы получим функцию $z(v), v \in\left[0, L_{2}\right]$. В частности, будет найдено значение $z(0)=L$. Непрерывный вид решения позволит построить обратную функцию $v(z), z \in\left[L_{1}, L\right]$ без потери точности. Таким образом, мы получим полное решение задачи (5.2)-(5.4). Отметим еще раз, что в рассмотренном случае $\lim _{z \rightarrow L} v^{\prime}(z)=-\infty$. Поэтому стандартное решение МГЭ в один этап невозможно, даже если мы знаем значение $L$.

Известно, что МГЭ редко применяется для решения одномерных задач. В данном случае это оправдывается получением непрерывного решения, что является преимуществом перед конечно-разностными методами, а также возможностью получения решения на заданном отрезке, что не всегда возможно методом степенных рядов. В совокупности с этими преимуществами, при условии достаточной точности, гранично-элементное решение представляется весьма перспективным. Сравнительная оценка точности решения различными методами задачи (5.1) в различных постановках при различных значениях параметров приведена в следующем разделе.

\section{6. Вычислительный эксперимент}

В данном разделе проведем сравнительный анализ приближенных решений задачи (5.2)-(5.4) в виде отрезков ряда (4.3), решений МГЭ и решений разностными методами. Последние будем строить следующим образом. Представим (5.2)-(5.4) в виде задачи Коши для системы уравнений первого порядка:

$$
\left\{\begin{array}{l}
v^{\prime}=w \\
w^{\prime}=\frac{1}{v} G(z, v, w)
\end{array} ; v(0)=0 ; w(0)=v_{*}^{\prime} .\right.
$$

Решать систему (6.1) будем методом Эйлера (МЭ) и методом Рунге-Кутта (МРК) четвертого порядка. Поскольку начальные условия заданы в точке сингулярности, на первом шаге в качестве решения примем решение в виде отрезка ряда, имеющего достаточно высокую точность вблизи начальной точки.

Проведенные исследования задачи (5.2)-(5.4) в различных постановках показали, что их решения можно условно разделить на следующие виды.

I. Ограниченные решения, определенные на отрезке $z \in[0, L]$, причем $v(L)>0$. Такой вид могут иметь решения задачи Коши (2.6), (2.7) (в этом случае $L=1$ ) и задачи (3.1), (3.2). Решение может быть как монотонно возрастающим на всем отрезке, так и иметь максимум $v_{\max }>0$ в некоторой точке $z_{\max } \in(0, L)$.

II. Ограниченные решения, определенные на отрезке $z \in[0, L]$, для которых $v(L)=0$, $\lim _{z \rightarrow L} v^{\prime}(z)=-\infty$. Решение имеет максимум $v_{\max }>0$ в точке $z_{\max } \in(0, L)$. Такой характер могут иметь решения как задачи (2.6), (2.7) (в этом случае $L<1$ ), так и задачи (3.1), (3.2).

III. Ограниченные решения задачи Коши (3.1), (3.2), определенные на бесконечном интервале $z \in[0,+\infty)$.

IV. Неограниченные монотонные решения $v(z)$, такие что $\lim _{z \rightarrow z_{*}} v(z)=+\infty$, где $z_{*}>0-$ конечно или бесконечно. Решения такого вида также характерны для бегущей волны.

Построение численного решения на всей области определения (отрезке) позволит получить глобальное эталонное решение уравнения (1.2). В случае неограниченной области определения мы можем построить численное решение лишь для ограниченного интервала времени. 


\section{1. Сравнение точных и приближенных решений}

В отдельных случаях удается получить точное решение задачи (5.2)-(5.4) в конечном виде. Эти решения дают возможность непосредственно оценить корректность приближенных решений.

Пример 1. В качестве начального примера рассмотрим задачу (2.6), (2.7), когда источник имеет вид $h(u)=u$, а закон движения фронта тепловой волны - показательный, $a(t)=e^{t}$. В этом случае в задаче $(5.1)$

$$
G\left(z, v, v^{\prime}\right)=(1-z) v^{\prime}-\frac{\left(v^{\prime}\right)^{2}}{\sigma}+v, v_{*}^{\prime}=\sigma
$$

Тогда точное решение задачи имеет вид:

$$
v(z)=\sigma z, z \in[0,1]
$$

и относится к виду 1 . Несмотря на простоту решения сравнение с ним позволяет сделать отдельные выводы о корректности приближенных методов. Очевидно, что решение методом степенных рядов, как и разностные методы, приведет к точному решению. Что касается МГЭ, то легко проверить, что представленный в разделе 5 алгоритм приведет к точному решению уже на первой итерации. Таким образом, уже на примере простого случая, приводящего к линейному решению, мы наблюдаем корректность предложенного алгоритма МГЭ.

Пример 2. Рассмотрим теперь более сложный случай, когда рассматриваемая задача Коши имеет точное аналитическое решение. На этот раз это будет задача (3.1), (3.2) при дробно-линейной зависимости коэффициента теплопроводности от температуры $\left(\kappa(T)=\frac{\lambda T}{T+C}\right)$ и при отсутствии источника $-h(u) \equiv 0$. Тогда $F(u)=\frac{\lambda+u}{\lambda-u}$ и в задаче $(5.1)$ :

$$
G\left(z, v, v^{\prime}\right)=\mu v^{\prime}-\frac{\lambda+v}{\lambda-v}\left(v^{\prime}\right)^{2}, v_{*}^{\prime}=\mu
$$

Точное решение задачи

$$
v(z)=\lambda\left(1-e^{-\frac{\mu z}{\lambda}}\right), z \in[0,+\infty)
$$

является монотонным и ограниченным и относится к виду III. Решение методом степенных рядов ввиду единственности аналитического решения приведет к ряду Тейлора для решения (6.5). Поэтому сравнивать будем точность решения МГЭ и разностными методами. В табл. 1 приведены погрешности полученных решений на отрезке $[0, L]$ при различных значениях параметров задачи. Параметр $h$ в случае разностных методов означает шаг интегрирования, а в случае МГЭ - расстояние между соседними точками коллокации, которые равномерно распределены на рассматриваемом отрезке.

Анализ полученных результатов показал следующее. При достаточно большом значении $h=0,1$ наибольшую точность имеют решения МГЭ. Сравнительно большая погрешность метода Рунге-Кутта четвертого порядка в этом случае обусловлена погрешностью на первом шаге, который мы делаем с помощью отрезка ряда. В проведенных расчетах мы использовали отрезки рядов десятой степени. В случае шага $h=0,01$ погрешность на первом шаге мала, вследствие чего точность метода Рунге-Кутта высока и превосходит другие методы. Таким 
образом, точность решения рассматриваемых задач разностными методами зависит от точности решения в виде отрезка ряда на первом шаге. В данном примере это отрезок ряда Тейлора точного решения (6.5), однако в общем случае радиус сходимости ряда (4.3), построение которого описано в разд. 4, неизвестен, поэтому точность решения непредсказуема.

Таблица 1 - Погрешности приближенных решений (пример 2)

\begin{tabular}{|c|c|c|c|c|c|c|}
\hline$\lambda$ & $\mu$ & $h$ & $L$ & МГЭ & MЭ & МРК \\
\hline 2 & 0,1 & 0,1 & 1 & $2,1 \cdot 10^{-5}$ & $2,9 \cdot 10^{-4}$ & $1,3 \cdot 10^{-4}$ \\
\hline 2 & 0,1 & 0,1 & 2 & $2,0 \cdot 10^{-5}$ & $5,0 \cdot 10^{-4}$ & $1,6 \cdot 10^{-4}$ \\
\hline 2 & 0,1 & 0,01 & 1 & $3,7 \cdot 10^{-7}$ & $2,4 \cdot 10^{-5}$ & $6,9 \cdot 10^{-11}$ \\
\hline 2 & 0,1 & 0,01 & 2 & $3,0 \cdot 10^{-7}$ & $4,5 \cdot 10^{-5}$ & $9,7 \cdot 10^{-10}$ \\
\hline 2 & 0,5 & 0,1 & 1 & $4,2 \cdot 10^{-4}$ & $5,8 \cdot 10^{-3}$ & $2,5 \cdot 10^{-3}$ \\
\hline 2 & 0,5 & 0,1 & 2 & $3,9 \cdot 10^{-4}$ & $8,3 \cdot 10^{-3}$ & $2,6 \cdot 10^{-3}$ \\
\hline 2 & 0,5 & 0,01 & 1 & $7,2 \cdot 10^{-6}$ & $4,8 \cdot 10^{-4}$ & $1,3 \cdot 10^{-9}$ \\
\hline 2 & 0,5 & 0,01 & 2 & $6,3 \cdot 10^{-6}$ & $7,6 \cdot 10^{-4}$ & $2,0 \cdot 10^{-9}$ \\
\hline 3 & 0,1 & 0,1 & 1 & $1,4 \cdot 10^{-5}$ & $1,9 \cdot 10^{-4}$ & $9,0 \cdot 10^{-5}$ \\
\hline 3 & 0,1 & 0,1 & 2 & $1,2 \cdot 10^{-5}$ & $3,4 \cdot 10^{-4}$ & $1,1 \cdot 10^{-4}$ \\
\hline 3 & 0,1 & 0,01 & 1 & $2,5 \cdot 10^{-7}$ & $1,6 \cdot 10^{-5}$ & $9,7 \cdot 10^{-11}$ \\
\hline 3 & 0,1 & 0,01 & 2 & $2,2 \cdot 10^{-7}$ & $3,1 \cdot 10^{-5}$ & $6,7 \cdot 10^{-10}$ \\
\hline 3 & 0,5 & 0,1 & 1 & $2,9 \cdot 10^{-4}$ & $4,2 \cdot 10^{-3}$ & $1,9 \cdot 10^{-3}$ \\
\hline 3 & 0,5 & 0,1 & 2 & $2,8 \cdot 10^{-4}$ & $6,6 \cdot 10^{-3}$ & $2,0 \cdot 10^{-3}$ \\
\hline 3 & 0,5 & 0,01 & 1 & $4,6 \cdot 10^{-6}$ & $3,5 \cdot 10^{-4}$ & $2,8 \cdot 10^{-10}$ \\
\hline 3 & 0,5 & 0,01 & 2 & $4,5 \cdot 10^{-6}$ & $5,9 \cdot 10^{-4}$ & $4,6 \cdot 10^{-10}$ \\
\hline
\end{tabular}

Отметим еще одно выявленное преимущество МГЭ перед разностными методами. При фиксированном шаге погрешность решения МГЭ не увеличивается, как у разностных решений, а уменьшается с увеличением интервала интегрирования. Это связано с увеличением числа точек коллокации.

\section{2. Оценка точности приближенных решений}

В следующих двух примерах точные решения в конечном виде найти не удалось, поэтому в качестве оценки точности приближенных решений принята невязка уравнения.

Пример 3. Рассмотрим задачу (2.6),(2.7) при степенном законе движения фронта тепловой волны вида $a(t)=(t+1)^{\frac{\beta-2}{2 \beta-2}}$, который соответствует источнику $h(u)=\alpha u^{\beta}$ при $\beta \neq 1,2$. Тогда в задаче (5.1)

$$
G\left(z, v, v^{\prime}\right)=(1-z) v^{\prime}-\frac{\left(v^{\prime}\right)^{2}}{\sigma}+\frac{2}{2-\beta} v-\alpha D v^{\beta} ; v_{*}^{\prime}=\sigma
$$

Решения этой задачи при $\sigma=3, \alpha=1$ и различных значениях $\beta$ методом граничных элементов представлены на рис. 1 . При $\beta=2.1,2.3$ областью определения решения является лишь часть отрезка $[0,1]: z \in[0, L], L<1, v(L)=0$. Эти решения относятся к виду II, и решения МГЭ строятся в два этапа, как описано в разд. 5. При больших значениях $\beta$ мы имеем решения вида I. 


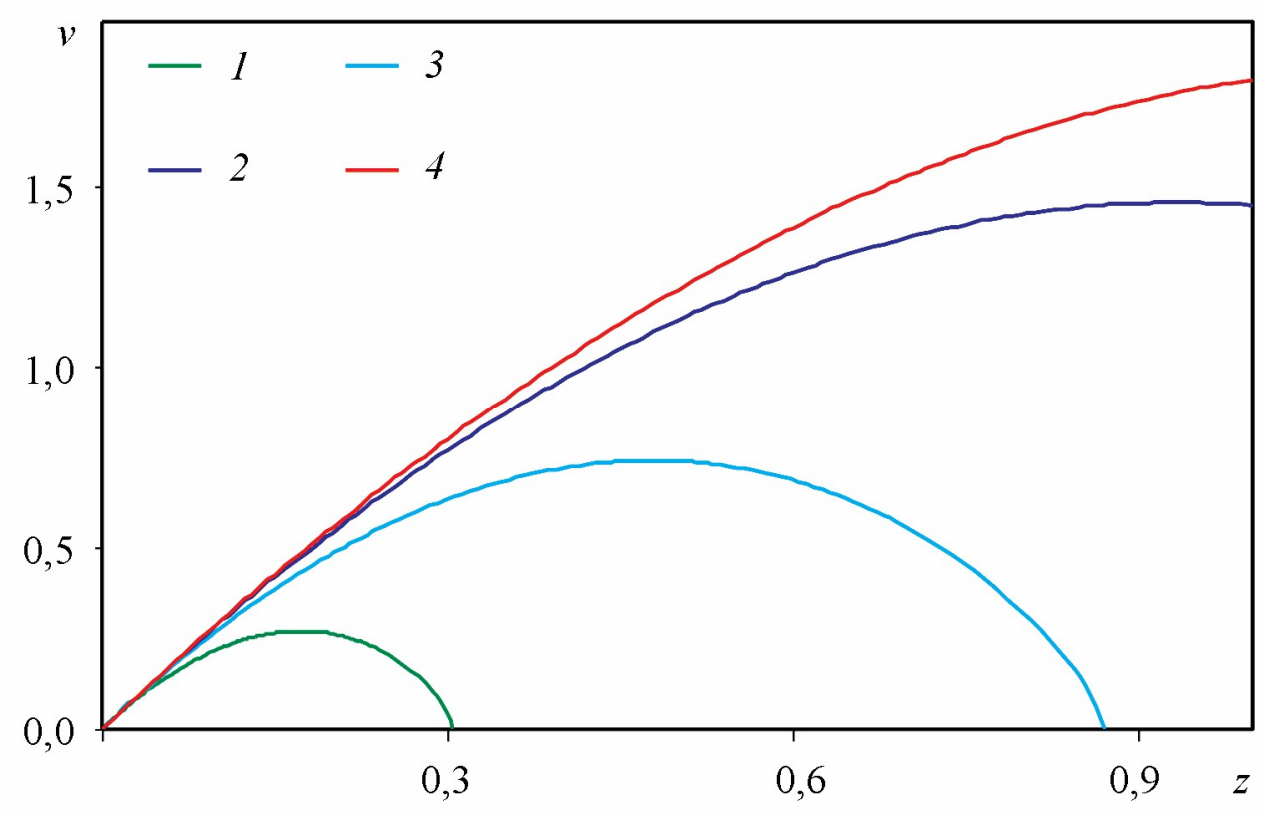

Рис. 1. Решения МГЭ (пример 3): $1-\beta=2,1 ; 2-\beta=2,7 ; 3-\beta=2,3 ; 4-\beta=3$

Непрерывный вид решений МГЭ позволяет дифференцировать их. Поэтому точность метода оценивалась подстановкой решения и его производных в решаемое уравнение $v v^{\prime \prime}-G\left(z, v, v^{\prime}\right)=0$. В качестве оценки точности было принято максимальное абсолютное значение левой части внутри интервала решения, т.е. невязка уравнения. В совокупности с выполнением начальных условий малое значение такой оценки показывает хорошую точность полученного решения. В табл. 2 приведем значения невязок для примера 2 при некоторых значениях параметров в сравнении с соответствующими погрешностями из табл. 1. Расчеты показывают хорошую корреляцию невязки и погрешности решений, что дает основания использовать невязку для оценки точности решения.

Таблица 2 - Невязки уравнения (3.1) для решений МГЭ (пример 2).

\begin{tabular}{|c|c|c|c|c|c|}
\hline$\lambda$ & $\mu$ & $h$ & $L$ & Невязка & Погрешность \\
\hline 2 & 0,1 & 0,1 & 2 & $2,1 \cdot 10^{-5}$ & $2,0 \cdot 10^{-5}$ \\
\hline 2 & 0,1 & 0,01 & 2 & $1,9 \cdot 10^{-7}$ & $3,0 \cdot 10^{-7}$ \\
\hline 2 & 0,5 & 0,1 & 2 & $2,8 \cdot 10^{-4}$ & $3,9 \cdot 10^{-4}$ \\
\hline 2 & 0,5 & 0,01 & 2 & $2,6 \cdot 10^{-5}$ & $6,3 \cdot 10^{-6}$ \\
\hline
\end{tabular}

Значения невязки уравнения (2.6) для примера 3 при $\sigma=3, \alpha=1$ представлены в табл. 3. Сравнительно большая невязка на втором этапе объясняется тем, что граничные условия на нем задаются по решению на первом этапе, т. е. уже с начальной погрешностью. Для сравнения приведем аналогичную оценку точности для решения в виде отрезка степенного ряда (4.3), который, отметим, мы можем построить только для целых значений $\beta$. При $\beta=3$ невязка уравнения (2.6) для отрезка ряда десятой степени составляет $1,5 \cdot 10^{-3}$, а для отрезка двадцатой степени $-8,2 \cdot 10^{-7}$, что хуже, чем для решения МГЭ.

Невозможность построения решения в виде ряда при нецелых значениях $\beta$ приводит к сложностям при решении разностными методами. В рассмотренных случаях для раскрытия особенности на первом шаге мы можем использовать лишь формулу Тейлора третьего порядка вместо отрезка ряда произвольной степени, это приводит к достаточно большой погрешности решения. Сравнение решения МГЭ с разностными решениями при целом значе- 
нии $\beta=3$ показано на рис. 2. Разностные решения приближаются к решению МГЭ с увеличением порядка точности. Это косвенно свидетельствует, что гранично-элементное решение не уступает в корректности разностным. Отметим, что решение методом Рунге-Кутта с шагом $h=0,01$ отличается от решения с шагом $h=0,05$ начиная с седьмого знака после запятой и не приведено на графике.

Таблица 3 - Невязки уравнения (2.6) для решений МГЭ (пример 3)

\begin{tabular}{|c|c|c|c|}
\hline$\beta$ & $h$ & Невязка на 1-м этапе & Невязка на 2-м этапе \\
\hline 2,1 & 0,05 & $7,6 \cdot 10^{-4}$ & $3,7 \cdot 10^{-4}$ \\
\hline 2,1 & 0,01 & $1,9 \cdot 10^{-5}$ & $2,9 \cdot 10^{-4}$ \\
\hline 2,3 & 0,05 & $6,7 \cdot 10^{-7}$ & $2,7 \cdot 10^{-4}$ \\
\hline 2,3 & 0,01 & $1,6 \cdot 10^{-7}$ & $1,1 \cdot 10^{-4}$ \\
\hline 2,7 & 0,05 & $2,1 \cdot 10^{-7}$ & - \\
\hline 2,7 & 0,01 & $3,4 \cdot 10^{-8}$ & - \\
\hline 3 & 0,05 & $1,0 \cdot 10^{-9}$ & - \\
\hline 3 & 0,01 & $8,2 \cdot 10^{-10}$ & - \\
\hline
\end{tabular}

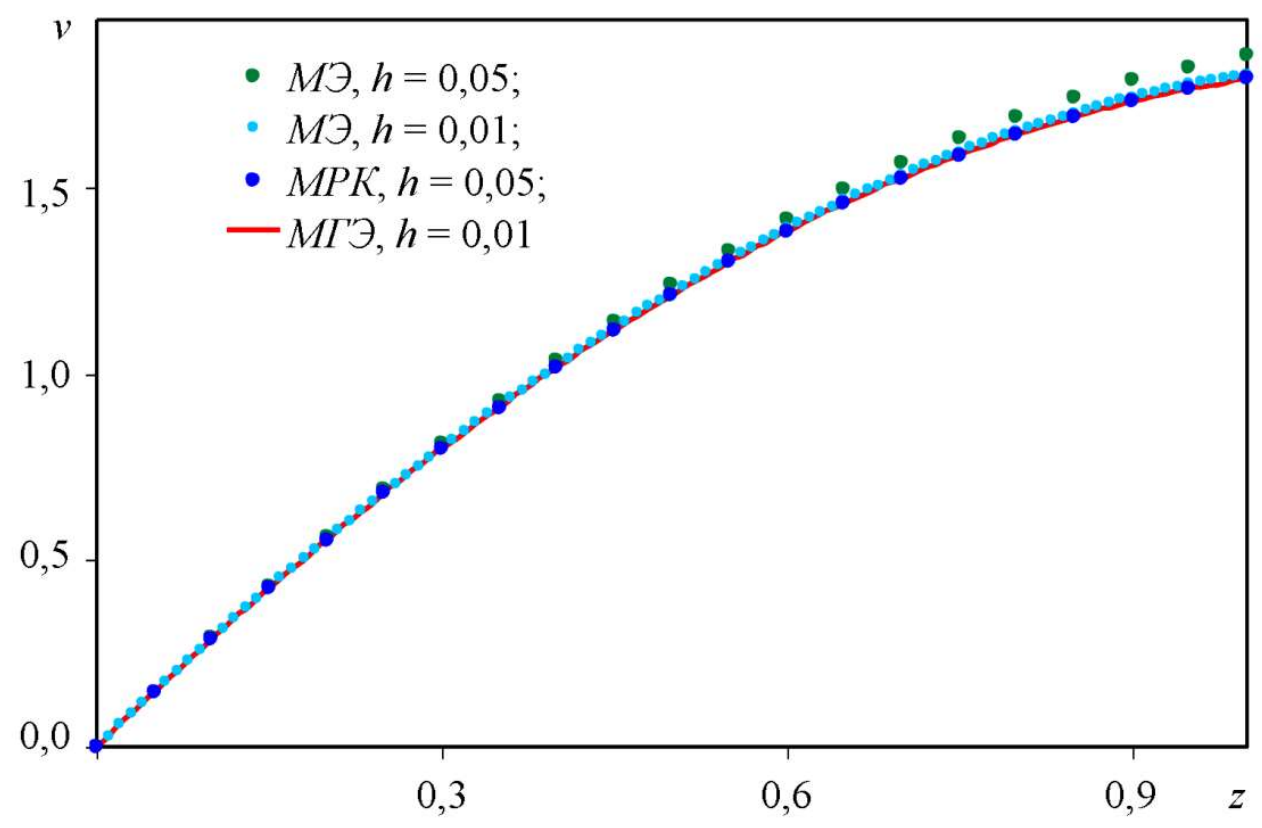

Рис. 2. Сравнение решения МГЭ и разностных решений при $\beta=3$ (пример 3)

Таким образом, пример 3 показал, что метод граничных элементов может быть применен в более широком диапазоне значений параметров, чем другие рассмотренные методы. Непрерывность полученного решения позволяет оценить точность выполнения решаемого уравнения. Сравнение с решениями другими методами показало меньшую погрешность решений МГЭ.

Пример 4. В завершение вычислительного эксперимента рассмотрим задачу, имеющую неограниченные решения вида IV. Именно, рассмотрим задачу (3.1), (3.2) для случая показательного коэффициента теплопроводности, $k(T)=\lambda\left(e^{C T}-1\right)$, в отсутствие источника, при $\alpha=0$. В этом случае 


$$
G\left(z, v, v^{\prime}\right)=\mu v^{\prime}-\frac{\lambda}{\lambda+v}\left(v^{\prime}\right)^{2}, v_{*}^{\prime}=\mu
$$

Оценку точности решения вновь будем проводить с помощью невязки уравнения (3.1). В табл. 4 приведены невязки решений на отрезке $z \in[0, L]$ для решений МГЭ при различных значениях $h$ расстояния между соседними точками коллокации, а также для отрезков ряда (4.3) различных степеней $n$. Полученные результаты показывают, что решение в виде ряда может иметь преимущество перед решением МГЭ, но при этом сильно зависит от параметров задачи. В свою очередь решения МГЭ слабо зависят от параметров и при этом дают невязки достаточно малого порядка. При этом порядок невязок для решений МГЭ не зависит от величины отрезка интегрирования в отличие от невязок для отрезков рядов.

Для сравнения решений МГЭ с разностными решениями было подсчитано их максимальное расхождение, которое наблюдалось на правом конце отрезка интегрирования. Результаты расчетов, приведенные в табл. 5, вновь показывают, что разностные решения приближаются к решению МГЭ с увеличением порядка точности метода. В качестве решений МГЭ здесь были приняты решения при $h=0,01$.

Таблица 4 - Невязки уравнения (3.1) для решений МГЭ и отрезков рядов (пример 3)

\begin{tabular}{|c|c|c|c|c|c|c|}
\hline \multirow{2}{*}{$\lambda$} & \multirow{2}{*}{$L$} & \multirow{2}{*}{$L$} & \multicolumn{2}{|c|}{ МГЭ } & \multicolumn{2}{c|}{ Ряд } \\
\cline { 4 - 7 } & & $h=0,1$ & $h=0,01$ & $n=5$ & $n=10$ \\
\hline 1 & 0,2 & 1 & $7,5 \cdot 10^{-6}$ & $1,1 \cdot 10^{-7}$ & $1,6 \cdot 10^{-7}$ & $5,1 \cdot 10^{-13}$ \\
\hline 1 & 0,2 & 2 & $3,5 \cdot 10^{-6}$ & $1,4 \cdot 10^{-7}$ & $5,0 \cdot 10^{-6}$ & $3,8 \cdot 10^{-10}$ \\
\hline 1 & 0,5 & 1 & $5,6 \cdot 10^{-5}$ & $1,8 \cdot 10^{-6}$ & $9,3 \cdot 10^{-5}$ & $2,2 \cdot 10^{-8}$ \\
\hline 1 & 0,5 & 2 & $3,4 \cdot 10^{-5}$ & $2,0 \cdot 10^{-6}$ & $2,7 \cdot 10^{-3}$ & $2,1 \cdot 10^{-5}$ \\
\hline 2 & 0,2 & 1 & $4,3 \cdot 10^{-6}$ & $4,0 \cdot 10^{-7}$ & $5,2 \cdot 10^{-9}$ & $2,6 \cdot 10^{-15}$ \\
\hline 2 & 0,2 & 2 & $3,7 \cdot 10^{-6}$ & $3,2 \cdot 10^{-7}$ & $1,6 \cdot 10^{-7}$ & $4,1 \cdot 10^{-13}$ \\
\hline 2 & 0,5 & 1 & $6,7 \cdot 10^{-5}$ & $6,0 \cdot 10^{-6}$ & $3,1 \cdot 10^{-6}$ & $2,9 \cdot 10^{-11}$ \\
\hline 2 & 0,5 & 2 & $5,9 \cdot 10^{-5}$ & $5,0 \cdot 10^{-6}$ & $9,3 \cdot 10^{-5}$ & $2,2 \cdot 10^{-8}$ \\
\hline
\end{tabular}

Таблица 5 - Максимальные отклонения разностных решений от решений МГЭ при $L=2$ (пример 4)

\begin{tabular}{|c|c|c|c|c|c|}
\hline \multirow{2}{*}{$\lambda$} & \multirow{2}{*}{$\mu$} & \multicolumn{2}{|c|}{ MЭ } & \multicolumn{2}{c|}{ MPК } \\
\cline { 3 - 6 } & & $h=0,1$ & $h=0,01$ & $h=0,1$ & $h=0,01$ \\
\hline 1 & 0,2 & $1,85 \cdot 10^{-3}$ & $1,92 \cdot 10^{-4}$ & $3,35 \cdot 10^{-6}$ & $3,32 \cdot 10^{-6}$ \\
\hline 1 & 0,5 & $1,07 \cdot 10^{-2}$ & $1,12 \cdot 10^{-3}$ & $2,74 \cdot 10^{-5}$ & $2,69 \cdot 10^{-5}$ \\
\hline 2 & 0,2 & $9,35 \cdot 10^{-4}$ & $9,28 \cdot 10^{-5}$ & $6,24 \cdot 10^{-6}$ & $6,23 \cdot 10^{-6}$ \\
\hline 2 & 0,5 & $5,68 \cdot 10^{-3}$ & $5,61 \cdot 10^{-4}$ & $4,51 \cdot 10^{-5}$ & $4,50 \cdot 10^{-5}$ \\
\hline
\end{tabular}

\section{7. Заключение}

В статье рассмотрена проблема нахождения точных решений для верификации приближенных методов построения тепловой волны, имеющей конечную скорость движения, для нелинейного уравнения теплопроводности с нелинейностью общего вида и источником. В условиях когда каких-либо строгих утверждений о сходимости приближенных методов доказать не удается, данная тематика приобретает большую актуальность. При этом построение точных решений сводится к интегрированию задач Коши для ОДУ второго порядка, имеющих особенность перед старшей производной, т. е. к необходимости решить задачу, хотя и более простую, чем исходная, но тоже нетривиальную. Предложены два способа по- 
строения: первый основан на методе степенных рядов, второй - на методе граничных элементов. Оба предполагают раскрытие особенности за счет специального выбора базисных функций, однако наборы последних различны, что позволяет, комбинируя подходы, добиваться хороших результатов. Сравнение решений двумя предложенными методами между собой, а также с разностными решениями методом Эйлера и методом Рунге-Кутта 4 порядка позволили сделать следующие основные выводы. В отдельных случаях решения методом степенных рядов и разностными методами имеют большую точность, чем решения МГЭ, а в других - меньшую. При этом решения МГЭ могут быть получены в более широком диапазоне параметров рассмотренных задач, их точность является удовлетворительной для поставленной задачи и в гораздо меньшей степени зависит от значений этих параметров, чем решения в виде ряда и разностные решения. Таким образом, МГЭ является среди использованных наиболее универсальным подходом к решению задач Коши рассмотренного типа для построения эталонных решений нелинейного вырождающегося уравнения теплопроводности. Дальнейшие исследования могут быть связаны с расширением класса используемых решений за счет применения обобщенных бегущих волн, а также с рассмотрением уравнения более общего вида, например содержащего конвективные слагаемые.

\section{Благодарность}

Работа выполнена при поддержке РФФИ, проект № 20-07-00407.

\section{Литература}

1. Vazquez J. L. The Porous Medium Equation: Mathematical Theory. - Oxford : Clarendon Press, 2007. - 648 p. - ISBN-10: 0198569033, ISBN-13: 978-019856903.

2. Режимы с обострением в задачах для нелинейных параболических уравнений / А. А. Самарский, В. А. Галактионов, С. П. Курдюмов, А. П. Михайлов - М. : Наука, 1987. $476 \mathrm{c}$.

3. Зельдович Я. Б., Компанеец А. С. К теории распространения тепла при теплопроводности, зависящей от температуры // В кн.: Сборник, посвященный 70-летию академика А. Ф. Иоффе. М. : Изд-во АН СССР, 1950. - С. 61-71.

4. Баренблатт Г. И., Вишик И. М. О конечной скорости распространения в задачах нестационарной фильтрации жидкости и газа // Прикладная математика и механика. - 1956. Т. 20, вып. 3. - С. 411-417.

5. Олейник О. А., Калашников А. С., Чжоу Юй-Линь. Задача Коши и краевые задачи для уравнений типа нестационарной фильтрации // Известия АН СССР. Серия математическая. 1958. - Т. 22, вып. 5. - С. 667-704.

6. Сидоров А. Ф. Избранные труды: Математика. Механика. - М. : Физматлит, 2001. 576 c. - ISBN 5-9221-0103-X.

7. Казаков А. Л., Лемперт А. А. Аналитическое и численное исследование одной краевой задачи нелинейной фильтрации с вырождением // Вычислительные технологии. - 2012. T. 17, № 1. - С. 57-68.

8. Казаков А. Л., Спевак Л. Ф. Методы граничных элементов и степенных рядов в одномерных задачах нелинейной фильтрации // Известия ИГУ. Серия Математика. - 2012. Т. 5, № 2. - C. 2-17.

9. Kazakov A. L., Spevak L. F. Numerical and analytical studies of a nonlinear parabolic equation with boundary conditions of a special form // Applied Mathematical Modelling. - 2013. Vol. 37, iss. 10-11. - P. 6918-6928. - DOI: 10.1016/j.apm.2013.02.026.

10. Казаков А. Л., Кузнецов П. А., Спевак Л. Ф. Об одной краевой задаче с вырождением для нелинейного уравнения теплопроводности в сферических координатах // Труды института математики и механики УрО РАН. - 2014. - Т. 20, № 1. - С. 119-129. 
11. Kazakov A. L., Spevak L. F. An analytical and numerical study of a nonlinear parabolic equation with degeneration for the cases of circular and spherical symmetry // Applied Mathematical Modelling. - 2016. - Vol. 40, iss. 2. - P. 1333-1343. - DOI: 10.1016/j.apm.2015.06.038.

12. Spevak L. F., Kazakov A. L. Constructing numerical solutions to a nonlinear heat conduction equation with boundary conditions degenerating at the initial moment of time // AIP Conference Proceedings. - 2016. - Vol. 1785, iss. 1. - P. 040076. - DOI: 10.1063/1.4967133.

13. Kazakov A. L., Kuznetsov P. A., Spevak L. F. A heat wave problem for a degenerate nonlinear parabolic equation with a specified source function // AIP Conference Proceedings. 2018. - Vol. 2053. - P. 030024. - DOI: 10.1063/1.5084385.

14. Казаков А. Л., Нефедова О. А., Спевак Л. Ф. Решение задач об инициировании тепловой волны для нелинейного уравнения теплопроводности методом граничных элементов // Журнал вычислительной математики и математической физики. $-2019 .-$ Т. 59, № 6. - C. 1047-1062. - DOI: 10.1134/S0044466919060085.

15. Казаков А. Л. О точных решениях краевой задачи о движении тепловой волны для уравнения нелинейной теплопроводности // Сибирские электронные математические известия. - 2019. - T. 16. - C. 1057-1068. - DOI: 10.33048/semi.2019.16.073. - URL: http://semr.math.nsc.ru/v16/p1057-1068.pdf

16. Spevak L. F., Kazakov A. L. Solving a degenerate nonlinear parabolic equation with a specified source function by the boundary element method // AIP Conference Proceedings. - 2017. Vol. 1915. - P. 040054. - DOI: 10.1063/1.5017402.

17. Kazakov A. L., Spevak L. F., Nefedova O. A. On the Numerical-Analytical Approaches to Solving a Nonlinear Heat Conduction Equation with a Singularity // Diagnostics, Resource and Mechanics of materials and structures. - 2018. - Iss. 6. - P. 100-116. - DOI: 10.17804/24109908.2018.6.100-116. - URL: http://dream-journal.org/issues/2018-6/2018-6_232.html

18. Banerjee P. K., Butterheld R. Boundary element methods in e- nineering science. US : McGraw-Hill Inc., 1981. - 452 p. - ISBN-10: 0070841209, ISBN-13: 978-0070841208.

19. Nardini D., Brebbia C. A. A New Approach to Free Vibration Analysis using Boundary Elements // Applied Mathematical Modelling. - 1983. - Vol. 7, no. 3. - P. 157-162. DOI: 10.1016/0307-904X(83)90003-3.

20. Wrobel L. C., Brebbia C. A. Nardini D. The dual reciprocity boundary element formulation for transient heat conduction // Finite elements in water resources VI. - Springer-Verlag : Berlin, Germany, 1986. - P. 801-811.

21. Tanaka M., Matsumoto T., Yang Q. F. Time-stepping boundary element method applied to 2-D transient heat conduction problems // Applied Mathematical Modelling. - 1994. - Vol. 18. P. 569-576. - DOI: 10.1016/0307-904X(94)90142-2.

22. Tanaka M., Matsumoto T., Takakuwa S. Dual reciprocity BEM for time-stepping approach to the transient heat conduction problem in nonlinear materials // Computer Methods in Applied Mechanics and Engineering. - 2006. - Vol. 195. - P. 4953-4961. - DOI: 10.1016/j.cma.2005.04.025.

23. Divo E., Kassab A. J. Transient non-linear heat conduction solution by a dual reciprocity boundary element method with an effective posteriori error estimator // Computers, Materials and Continua. - 2005. - Vol. 2. - P. 277-288. - DOI: 10.3970/cmc.2005.002.277.

24. Wrobel L. C., Brebbia C. A. The dual reciprocity boundary element formulation for nonlinear diffusion problems // Computer Methods in Applied Mechanics and Engineering. - 1987. Vol. 65. - P. 147-164. - DOI: 10.1016/0045-7825(87)90010-7.

25. Singh K. M., Tanaka M. Dual reciprocity boundary element analysis of transient advectiondiffusion // International Journal of Numerical Methods for Heat \& Fluid Flow. - 2003. - Vol. 13. P. 633-646. - DOI: 10.1108/09615530310482481.

26. Al-Bayati S. A., Wrobel L. C. A novel dual reciprocity boundary element formulation for two-dimensional transient convection-diffusion-reaction problems with variable 
velocity // Engineering Analysis with Boundary Elements. - 2018. - Vol. 94. - P. 60-68. DOI: 10.1016/j.enganabound.2018.06.001.

27. Fendoglu H., Bozkaya C., Tezer-Sezgin M. DBEM and DRBEM solutions to 2D transient convection-diffusion-reaction type equations // Engineering Analysis with Boundary Elements. 2018. - Vol. 93. - P. 124-134. - DOI: 10.1016/j.enganabound.2018.04.011.

28. Powell M. J. D. The Theory of Radial Basis Function Approximation // Light W., ed. Advances in Numerical Analysis. - Oxford, UK : Oxford Science Publications, 1992. - Vol. 2.

29. Golberg M. A., Chen C. S., Bowman H. Some recent results and proposals for the use of radial basis functions in the BEM // Engineering Analysis with Boundary Elements. - 1999. Vol. 23. - P. 285-296. - DOI: 10.1016/S0955-7997(98)00087-3.

30. Kazakov A. L., Kuznetsov P. A., Spevak L. F. Analytical and numerical construction of heat wave type solutions to the nonlinear heat equation with a source // Journal of Mathematical Sciences. - 2019. - Vol. 239, no. 2. - P. 111-122. - DOI: 10.1007/s10958-019-04294-x.

31. Kazakov A. L., Spevak L. F. Numerical Study of Travelling Wave Type Solutions for the Nonlinear Heat Equation // AIP Conference Proceedings. - 2019. - Vol. 2176. - P. 030006. DOI: $10.1063 / 1.5135130$.

32. Полянин А. Д., Зайцев В. Ф., Журов А. И. Нелинейные уравнения математической физики и механики. Методы решения. - М. : Изд-во Юрайт, 2017. - 256 с. - ISBN 978-5-534-02317-6. 